\title{
Ultrafast dynamical path for the switching of a ferrimagnet after femtosecond heating
}

\author{
U. Atxitia, ${ }^{1}$ T. Ostler, ${ }^{1}$ J. Barker, ${ }^{1}$ R. F. L. Evans, ${ }^{1}$ R. W. Chantrell, ${ }^{1}$ and O. Chubykalo-Fesenko ${ }^{2}$ \\ ${ }^{1}$ Department of Physics, University of York, Heslington, York YO10 5DD United Kingdom \\ ${ }^{2}$ Instituto de Ciencia de Materiales de Madrid, CSIC, Cantoblanco, 28049 Madrid, Spain
}

(Received 17 July 2012; revised manuscript received 6 December 2012; published 20 June 2013)

\begin{abstract}
Ultrafast laser-induced magnetic switching in rare earth-transition metal ferrimagnetic alloys has recently been reported to occur by ultrafast heating alone. Using atomistic simulations and a ferrimagnetic Landau-LifshitzBloch formalism, we demonstrate that for switching to occur it is necessary that angular momentum is transferred from the longitudinal to transverse magnetization components in the transition metal. This dynamical path leads to the transfer of the angular momentum to the rare earth metal and magnetization switching with subsequent ultrafast precession caused by the intersublattice exchange field on the atomic scale.
\end{abstract}

DOI: 10.1103/PhysRevB.87.224417

PACS number(s): 75.78.Jp, 75.10.Hk, 75.50.Gg

The behavior of magnetization dynamics triggered by an ultrafast laser stimulus is a topic of intense research interest in both fundamental and applied magnetism. ${ }^{1}$ A range of studies using ultrafast laser pulses have shown very different time scales of demagnetization for different materials; from $100 \mathrm{fs}$ in $\mathrm{Ni}^{2}$ to $100 \mathrm{ps}$ in $\mathrm{Gd}^{3}$ Any potential applications utilizing such a mechanism would require, not only ultrafast demagnetization, but also controlled magnetization switching.

Magnetization reversal induced by an ultrafast laser pulse has been reported in the ferrimagnet $\mathrm{GdFeCo}$, together with a rich variety of phenomena. ${ }^{4-8}$ Several hypotheses have been put forward to explain the observed magnetization switching: crossing of the angular momentum compensation point, ${ }^{4}$ the Inverse Faraday Effect, ${ }^{5}$ and its combination with ultrafast heating. ${ }^{6}$ It has been shown that the rare earth (RE) responds more slowly to the laser pulse than the transition metal (TM), ${ }^{7}$ even though the sublattices are strongly exchange coupled. Intriguingly, Radu et $a .^{7}$ show experimentally and theoretically the existence of a transient ferromagnetic-like state, whereby the two sublattices align against their exchange interaction, existing for a few hundred femtoseconds. Recently, the atomistic model outlined in Refs. 7 and 8 predicted the phenomenon of magnetization reversal induced by heat alone, in the absence of any external field; a prediction verified experimentally. This remarkable result opens many interesting possibilities in terms of ultrafast magnetization reversal and potential areas of practical exploitation, however a complete theoretical understanding of this effect is currently missing.

In magnets consisting of more than one magnetic species, excitation of the spins on a time scale comparable with that of the intersublattice exchange takes the sublattices out of equilibrium with each other. It is in this regime where the thermally driven switching of ferrimagnetic GdFeCo occurs. A recent study by Mentink et al. ${ }^{9}$ proposed an explanation of the process using a phenomenological model of the magnetization dynamics, which assumes the additive character of two relaxation mechanisms: one governed by the intersublattice exchange and another by the relativistic contribution (coupling to external degrees of freedom). The model is based on the physically plausible argument that the switching is driven by angular momentum transfer in the exchange-dominated regime. However, the assumption of a linear path to reversal allows the angular momentum transfer to occur through longitudinal components only, since the perpendicular components are neglected. Additionally, the dynamical equation in Ref. 9 was derived from the Onsager principle, generally valid for small deviations from the equilibrium only. Thus far, a complete explanation of the heat-driven, ultrafast reversal process remains illusive.

In this article we demonstrate that the switching of magnetization in a ferrimagnet after femtosecond heating is due to the transfer of angular momentum from the longitudinal to the transverse magnetization components in the TM and consequent transfer of the angular momentum through perpendicular components to the RE. We use the Landau-LifshitzBloch (LLB) macroscopic equation of motion for amorphous ferrimagnetic alloys ${ }^{10}$ in which, unlike the phenomenological model of Ref. 9, the two relaxation mechanisms are not additive. Our theory gives the nonequilibrium conditions necessary for this angular momentum transfer to happen and thus to produce the precessional rather than a linear route to magnetization reversal as suggested in Ref. 9.

In the absence of any external stimulus, the energetics of the atomistic spin model are described purely by exchange interactions, given by the spin Hamiltonian:

$$
\mathcal{H}=-\sum_{j<i} J_{i j} \mathbf{S}_{i} \cdot \mathbf{S}_{j}
$$

where $J_{i j}$ is the exchange integral between spins $i$ and $j(i, j$ are lattice sites), and where $j$ runs over first nearest neighbors only, $\mathbf{S}_{i}$ is the normalized magnetic moment $\left|\mathbf{S}_{i}\right|=1$. We model the magnetization dynamics of the system using the LandauLifshitz-Gilbert (LLG) equation with Langevin dynamics, as detailed in Ref. 11.

We begin by considering our simple spin model with a structure similar to a magnetic dot like the ones used in bit-patterned media. The dimensions of the dot are $40 \mathrm{~nm} \times 40 \mathrm{~nm} \times 5 \mathrm{~nm}$ with $25 \% \mathrm{Gd}$. To simulate the effect of an ultrafast heat pulse we simulate a steplike temperature pulse of duration $600 \mathrm{fs}$ with a value of $T=1000 \mathrm{~K}$. This pulse height is just above the pulse power threshold for switching. Figure 1 shows the magnetization dynamics of the transition metal sublattice just after the action of the pulse. The results show that after the pulse, large amplitude precession is induced. This onset of precession as we cross the boundary to switching, as we will show, provides an important insight into the mechanism for the heatinduced switching in Ref. 8. Figure 2 shows the magnetization 


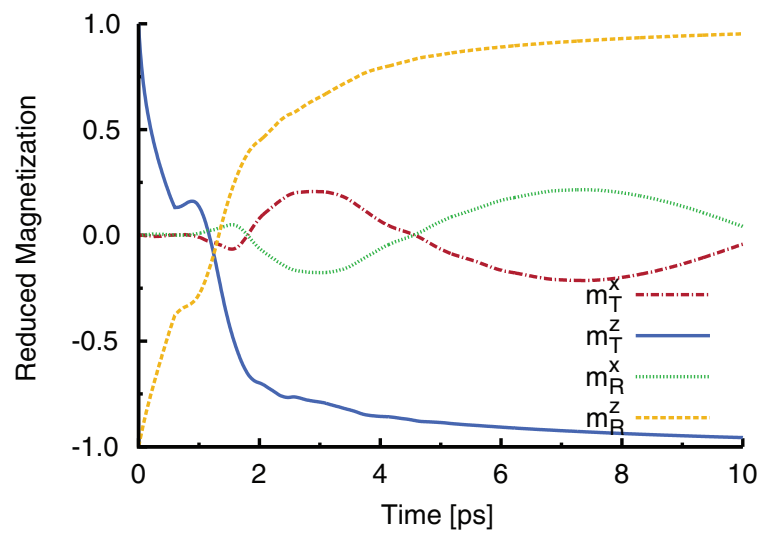

FIG. 1. (Color online) Atomistic computer simulations of laser induced magnetization reversal in a $40 \times 40 \times 5 \mathrm{~nm}^{3}$ size GdFeCo ferrimagnetic dot. The $z$ component of each sublattice shows that the reversal takes place, while the $x$ component shows that a magnetic precession is developed.

dynamics for a range of peak temperatures from just above the threshold switching temperature to high temperature. The $z$ and transverse component of the magnetization dynamics shown in panels (a) and (b). Panel (c) shows the peak transverse component as a function of the temperature pulse height, $T_{\max }$. The induced precession on the boundary of switching provides a challenging experiment to verify the theory that we present below. While atomistic models have proven to be a powerful tool in predicting heat-induced switching, ${ }^{7,8}$ they fail to provide a simple picture for the cause of its physical origin. However, the macroscopic LLB equation has been demonstrated to be an adequate approach, allowing a simple description of ultrafast magnetization phenomena, ${ }^{12,13}$ but until recently it existed only for a single species ferromagnet. ${ }^{14}$
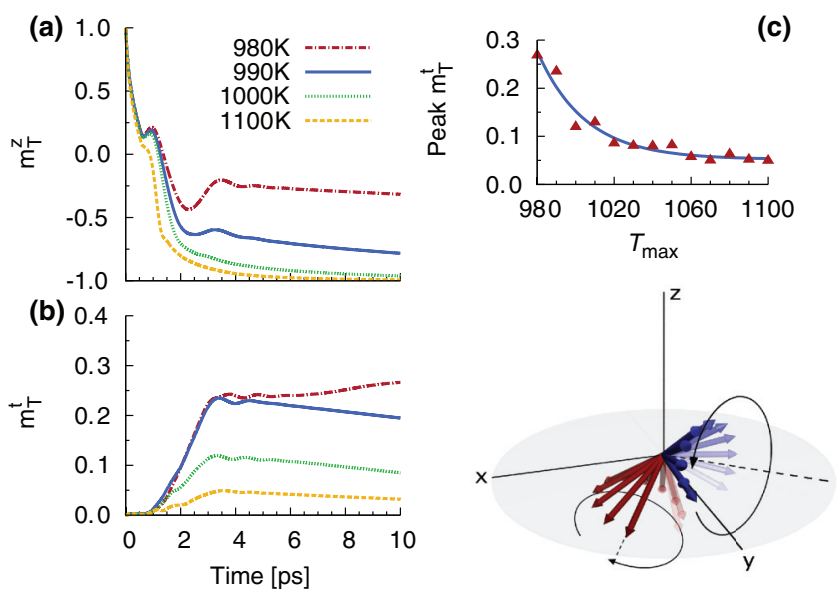

FIG. 2. (Color online) Magnetization dynamics for a range of pulse temperatures, $T_{\max }$. Panels (a) and (b) show the $z$ component, and magnitude of the transverse component respectively. Panel (c) shows the peak value of the transverse component as a function of the pulse temperature, points represent the data and the line is a guide to the eye. The schematic shows what we observe from the model, namely, a mutual precessional motion due to the exchange field exerted by the opposite sublattice with high frequency precessional motion towards the direction of the other sublattice.
Recently ${ }^{10}$ we have derived the LLB equation for a two species system which describes the average magnetization dynamics in each sublattice $\mathbf{m}_{v}=\left\langle\mathbf{S}_{i}^{v}\right\rangle$, where $v$ stands for TM or RE sublattice in this case and $i$ for spins in the sublattice $v$. Importantly, unlike the approach used in Ref. 9, the derivation does not use the Onsager principle and is thus valid far from equilibrium. In the absence of an applied field and anisotropy, the LLB equation for the TM is written as

$$
\frac{1}{\left|\gamma_{\mathrm{T}}\right|} \frac{d \mathbf{m}_{\mathrm{T}}}{d t}=-\mathbf{m}_{\mathrm{T}} \times\left[\mathbf{H}_{\mathrm{T}}^{\mathrm{EX}}+\frac{\alpha_{\mathrm{T}}^{\perp}}{m_{\mathrm{T}}^{2}} \mathbf{m}_{\mathrm{T}} \times \mathbf{H}_{\mathrm{T}}^{\mathrm{EX}}\right]+\alpha_{\mathrm{T}}^{\|} H_{\mathrm{T}}^{\|} \mathbf{m}_{\mathrm{T}},
$$

with a complementary equation for the RE. The exchange field from the RE is calculated via the mean-field approximation (MFA) of the impurity model presented in Ref. 11 as $\mathbf{H}_{\mathrm{T}}^{\mathrm{EX}}=\left(J_{0, \mathrm{TR}} / \mu_{\mathrm{T}}\right) \mathbf{m}_{\mathrm{R}}$, where $J_{0, \mathrm{TR}}=x z J_{\mathrm{TR}}, x$ is the impurity content, $z$ the number of nearest TM neighbors in the ordered lattice, and $J_{\mathrm{TR}}<0$ the intersublattice exchange parameter. The TM magnetic moment is denoted $\mu_{T}, \gamma_{\mathrm{T}}$ is the gyromagnetic ratio for the TM lattice, and $\alpha_{\mathrm{T}}^{\|}(T)$ and $\alpha_{\mathrm{T}}^{\perp}(T)$ are temperature-dependent TM longitudinal and transverse damping parameters, linearly proportional to the intrinsic coupling to the bath parameter $\lambda_{\mathrm{T}} .{ }^{10}$ The longitudinal effective field in Eq. (2) reads

$$
H_{\mathrm{T}}^{\|}=\frac{\Gamma_{\mathrm{TT}}}{2}\left(1-\frac{m_{\mathrm{T}}^{2}}{m_{e, \mathrm{~T}}^{2}}\right)-\frac{\Gamma_{\mathrm{TR}}}{2}\left(1-\frac{\tau_{\mathrm{R}}^{2}}{\tau_{e, \mathrm{R}}^{2}}\right),
$$

where $\tau_{\mathrm{R}}=\left|\left(\mathbf{m}_{\mathrm{T}} \cdot \mathbf{m}_{\mathrm{R}}\right)\right| / m_{\mathrm{T}}$ is the absolute value of the projection of the RE magnetization onto the TM magnetization and $\tau_{e, \mathrm{R}}$ is its equilibrium value. The rate parameters in Eq. (3) read

$\Gamma_{\mathrm{TT}}=\frac{1}{\tilde{\chi}_{\mathrm{T}, \|}}\left(1+\frac{\left|J_{0, \mathrm{TR}}\right|}{\mu_{\mathrm{T}}} \tilde{\chi}_{\mathrm{R}, \|}\right), \quad \Gamma_{\mathrm{TR}}=\frac{\left|J_{0, \mathrm{TR}}\right|}{\mu_{\mathrm{T}}} \frac{\tau_{e, \mathrm{R}}}{m_{e, \mathrm{~T}}}$.

They are temperature-dependent via the equilibrium magnetizations and partial longitudinal susceptibilities $\tilde{\chi}_{\mathrm{T}, \|}=$ $\left(\partial m_{\mathrm{T}} / \partial H\right)_{H \rightarrow 0}, \quad \tilde{\chi}_{\mathrm{R}, \|}=\left(\partial m_{\mathrm{R}} / \partial H\right)_{H \rightarrow 0}$, evaluated in the MFA in the presence of intersublattice and intrasublattice exchange. ${ }^{10}$

In Eq. (2) the first term in the right-hand side describes the precession of the TM magnetization, $\mathbf{m}_{\mathrm{T}}$, around the exchange field produced by the RE sublattice. Although this term conserves the magnetization modulus, $m_{\mathrm{T}}$, it allows transfer of angular momentum between lattices. The second term in Eq. (2) describes the relaxation of $\mathbf{m}_{\mathrm{T}}$ towards the antiparallel alignment between both sublattice magnetizations. Finally, the third term in Eq. (2) defines the longitudinal relaxation, comprising the difference between relaxation coming from the deviations of TM magnetization from equilibrium and those of RE. In the ferrimagnetic LLB all three terms act on the time scale given by the exchange interactions in comparison to the ferromagnetic LLB case, where the longitudinal and transverse motion have very different time scales. ${ }^{15,16}$

Figure 3 shows the direct numerical integration of Eq. (2). With initial antiparallel alignment of the RE and TM, $\mathbf{m}_{\mathrm{T}} \| \mathbf{m}_{\mathrm{R}}$, when the temperature is raised both sublattice magnetizations are reduced, followed by the linear magnetization recovery path to the expected ground state (see dashed lines in Fig. 3) and does not produce switching. In this case no torque is exerted from one sublattice to another as $\mathbf{m}_{\mathrm{T}} \times \mathbf{m}_{\mathrm{R}}=0$. 


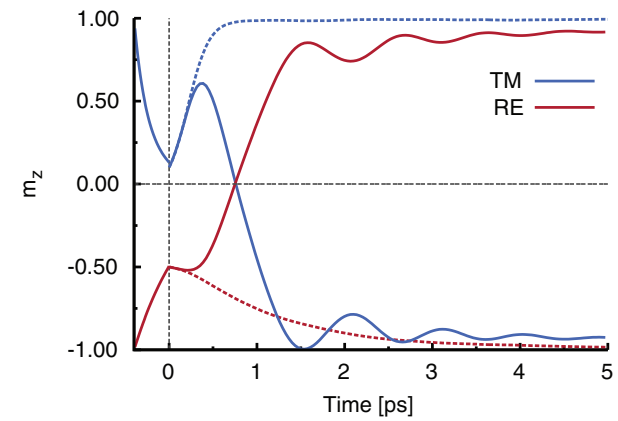

FIG. 3. (Color online) Numerical integration of the switching behavior for the nonstochastic LLB with a small angle $\left(15^{\circ}\right)$ between sublattices (solid lines). Without the angle (dashed lines) switching does not occur, as predicted. The time $t=0$ corresponds to the end of the square shaped laser pulse with $T_{\max }=1500 \mathrm{~K}$. For the integration at temperatures above $T_{C}$ we use the paramagnetic version of the ferrimagnetic LLB equation with MFA. ${ }^{10}$

However, this torque, which allows transfer of angular momentum between sublattices, is always present in the full atomistic approach with stochastic fields because of the high temperatures reached during the reversal process. We can include in Eq. (2) the presence of this torque by canting by a small angle the two sublattices magnetization once the heat pulse is gone or alternatively by the integration of the stochastic LLB equation. ${ }^{17}$ The solid lines in Fig. 3 show the integration of Eq. (2) including this angle and shows reversal. This small angle generates a mutual precessional motion which occurs due to the exchange field exerted by the opposite sublattice and the transverse relaxation directed towards the direction of the opposite sublattice. This mutual motion leads to the switching, as illustrated in the schematic of Fig. 2.

Though the longitudinal magnetization process contributes to the time scale of reversal it does not drive the switching process. Unlike the statement in Ref. 9, the longitudinal relaxation itself cannot change the direction of $\mathbf{m}_{\mathrm{T}}$, due to the multiplication of the longitudinal relaxation term in Eq. (2) by $\mathbf{m}_{\mathrm{T}}$. In order to understand the switching mechanism we therefore need to consider both longitudinal and transverse relaxation.

Now we demonstrate that at high temperatures the longitudinal relaxation becomes unstable. This happens because close to $T_{c}$ the sign of $H_{\mathrm{T}}^{\|}$can change. In Fig. 4 we present the temperature dependence of relaxation rates (4) evaluated for the parameters of $\mathrm{GdFeCo}^{11}$ in the MFA. One can see that close to $T_{c}: \Gamma_{\mathrm{TT}}<\Gamma_{\mathrm{TR}}$. Firstly we reduce the LLB equation (2) to a dynamical system, based on information from atomistic modeling. We can assume that slightly before the reversal the initial transverse magnetization components of the sublattices are small (but not zero), and that the modulus of the TM sublattice is much smaller than that of the RE $\left(m_{\mathrm{T}}^{z} \ll m_{\mathrm{R}}^{0}\right)$, owing to the faster relaxation time of the TM. In this approximation the longitudinal field is positive $H_{\mathrm{T}}^{\|}>0: H_{\mathrm{T}}^{\|} \simeq \Gamma_{\mathrm{TR}} \frac{m_{\mathrm{R}}^{0}}{m_{\mathrm{R}}^{e}}$ for the case before the heat pulse is removed $\left(m_{\mathrm{T}}>m_{e, \mathrm{~T}}=0\right)$ and because after the heat pulse is gone the system cools down $H_{\mathrm{T}}^{\|} \simeq\left[\Gamma_{\mathrm{TT}}-\Gamma_{\mathrm{TR}}\right] / 2>0$ with $m_{\mathrm{T}} \ll m_{e, \mathrm{~T}}(T)$. The LLB equation for the TM is reduced to

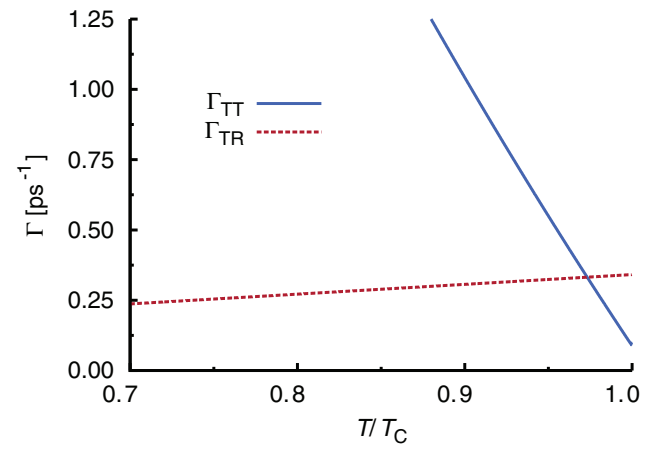

FIG. 4. (Color online) Longitudinal relaxation rates as a function of temperature in the LLB equation, evaluated for GdFeCo parameters. The dashed line shows the TM-RE relaxation rate and the solid line is that of the TM-TM interaction. At low temperatures $\Gamma_{\mathrm{TT}} \gg$ $\Gamma_{\mathrm{TR}}$, due to the small value of the susceptibility $\tilde{\chi}_{\mathrm{T}, \|}$, therefore the relaxation of the TM magnetization is always to its own equilibrium. However, at temperatures close to $T_{c}, \Gamma_{\mathrm{TT}}<\Gamma_{\mathrm{TR}}$, thus the TM prefers to relax towards the RE magnetization in this regime.

the following system of equations:

$$
\begin{aligned}
\frac{d m_{\mathrm{T}}^{2}}{\mathrm{~d} t} & =2\left|\gamma_{\mathrm{T}}\right| \alpha_{\mathrm{T}}^{\|} H_{\mathrm{T}}^{\|} m_{\mathrm{T}}^{2}, \\
\frac{d \rho}{d t} & =-2\left[\alpha_{\mathrm{T}}^{\perp} \Omega_{\mathrm{T}} \sqrt{1-\rho / m_{\mathrm{T}}^{2}}-\left|\gamma_{\mathrm{T}}\right| \alpha_{\mathrm{T}}^{\|} H_{\mathrm{T}}^{\|}\right] \rho,
\end{aligned}
$$

where $\rho=\left(m_{\mathrm{T}}^{t}\right)^{2}=\left(m_{\mathrm{T}}^{x}\right)^{2}+\left(m_{\mathrm{T}}^{y}\right)^{2}$ is the TM transverse magnetization component, $\Omega_{\mathrm{T}}=m_{\mathrm{R}}^{0}\left|\gamma_{\mathrm{T}}\right|\left|J_{0, \mathrm{TR}}\right| / \mu_{\mathrm{T}}$ is the precessional frequency of the anti-ferromagnetic exchange mode.

The trajectory $\rho=0$ corresponds to a linear dynamical mode. The standard analysis of the dynamical system (5) shows that for $H_{\mathrm{T}}^{\|}>0$ and $m_{\mathrm{T}}^{z}<\alpha_{\mathrm{T}}^{\perp} \Omega_{\mathrm{T}} /\left(\left|\gamma_{\mathrm{T}}\right| H_{\mathrm{T}}^{\|}\right)$this trajectory becomes unstable. Before the end of the pulse it is equivalent to $m_{\mathrm{T}}>\left(\alpha_{\mathrm{T}}^{\perp} / \alpha_{\mathrm{T}}^{\|}\right) m_{e, \mathrm{~T}}$, which is also easily satisfied, taking into account that $\alpha_{\mathrm{T}}^{\perp}>\alpha_{\mathrm{T}}^{\|}$; see Ref. 10. The physical interpretation is that in this case very small perturbations from $\rho=0$ will not be damped but will lead to the development of a perpendicular magnetization component, as is indeed observed by the atomistic simulations in Fig. 1.

However, the dynamical system (5) alone does not describe the reversal due to the assumption of the static RE magnetization. In the same approximation, the LLB equation for the RE reads

$\frac{d m_{\mathrm{R}}^{x(y)}}{d t}= \pm \Omega_{\mathrm{R}} m_{\mathrm{T}}^{y(x)}-\frac{\alpha_{\mathrm{R}}^{\perp}}{m_{\mathrm{R}}^{0}} \Omega_{\mathrm{R}} m_{\mathrm{T}}^{x(y)}-\left|\gamma_{\mathrm{R}}\right| \alpha_{\mathrm{R}}^{\|} H_{\mathrm{R}}^{\|} m_{\mathrm{R}}^{x(y)}$,

where the upper sign corresponds to the equation for $m_{\mathrm{R}}^{x}$ and the lower sign for the $m_{\mathrm{R}}^{y}$ one, $\Omega_{\mathrm{R}}=z q m_{\mathrm{R}}^{0}\left|\gamma_{\mathrm{R}}\right|\left|J_{\mathrm{TR}}\right| / \mu_{\mathrm{R}}$ and $H_{\mathrm{R}}^{\|}$is the RE longitudinal field. Equation (6) shows that the perpendicular motion of the TM triggers the corresponding precessional motion of the RE via the angular momentum transfer [the first two terms of Eq. (2), i.e., via perpendicular components] with the same frequency $\Omega_{\mathrm{T}}$, but different amplitude; see the schematic in Fig. 2. During this dynamical process in some time interval the RE and TM magnetization have both the same sign of the $z$ component, forming the transient ferromagnetic-like state seen experimentally. ${ }^{7}$ Note 
that the subsequent precession has a frequency which is proportional to the exchange field and thus is extremely fast. The motion of the TM around RE direction and vice versa occurs during and after the ferromagnetic-like state until the system has relaxed to equilibrium.

An outstanding question is whether the magnetization precession, a central part of the process, can be observed experimentally on a macroscopic sample. We should recall that in nonequilibrium at high temperatures the correlation between atomic sites is weak, thus we cannot expect the precession to occur with the same phase in the whole sample, an effect which would make the precession macroscopically unobservable. Usually in large system sizes $\left[>(20 \mathrm{~nm})^{3}\right]$ precession is averaged out, consistent with the excitation of localized exchange modes with random phase. Note that the same effect happens for very high temperatures where the observed magnetization trajectory appears close to linear, although we stress again the importance of a small perpendicular component to initiate the magnetization reversal.

In conclusion, the LLB equation for a ferrimagnet describes the mutual relaxation of sublattices which occurs simultaneously under internal damping and intersublattice exchange. This model allows us to present a simple picture of the magnetization reversal of GdFeCo in response to an ultrafast heat pulse alone. The physical origin of this effect is revealed within the LLB equation as a dynamical reversal path resulting from the instability of the linear motion. To trigger the reversal path a small perpendicular component is necessary. In practice this will arise from random fluctuations of the magnetization at elevated temperatures. The perpendicular component grows in time resulting in ultrafast magnetization precession in the intersublattice exchange field, also observed in atomistic simulations for small system sizes. The switching is initiated by the TM, which arrives at zero magnetization faster than the RE and responds dynamically to its exchange field. Thus, the nonequivalence of the two sublattices is an essential part of the process. Switching into the transient ferromagnetic state occurs due to large-amplitude precessional motion of the TM in the exchange field from the RE and a slow dynamics of RE.

This work was supported by the European Community's Seventh Framework Programme (FP7/2007-2013) under grant agreements NMP3-SL-2008-214469 (UltraMagnetron) N 214810 (FANTOMAS), and NMP3-SL-2012281043 (FEMTOSPIN) and the Spanish Ministry of Science and Innovation under the grant FIS2010-20979-C02-02.
${ }^{1}$ J. Stöhr and H. C. Siegmann, Magnetism: From Fundamentals to Nanoscale Dynamics (Springer, Berlin, 2006).

${ }^{2}$ E. Beaurepaire, J.-C. Merle, A. Daunois, and J.-Y. Bigot, Phys. Rev. Lett. 76, 4250 (1996).

${ }^{3}$ M. Wietstruk, A. Melnikov, C. Stamm, T. Kachel, N. Pontius, M. Sultan, C. Gahl, M. Weinelt, H. A. Dürr, and U. Bovensiepen, Phys. Rev. Lett. 106, 127401 (2011).

${ }^{4}$ C. D. Stanciu, F. Hansteen, A. V. Kimel, A. Kirilyuk, A. Tsukamoto, A. Itoh, and T. Rasing, Phys. Rev. Lett. 99, 047601 (2007).

${ }^{5}$ F. Hansteen, A. Kimel, A. Kirilyuk, and T. Rasing, Phys. Rev. Lett. 95, 047402 (2005).

${ }^{6}$ K. Vahaplar, A. M. Kalashnikova, A. V. Kimel, D. Hinzke, U. Nowak, R. Chantrell, A. Tsukamoto, A. Itoh, A. Kirilyuk, and T. Rasing, Phys. Rev. Lett. 103, 117201 (2009).

${ }^{7}$ I. Radu, K. Vahaplar, C. Stamm, T. Kachel, N. Pontius, H. A. Dürr, T. A. Ostler, J. Barker, R. F. L. Evans, R. W. Chantrell, A. Tsukamoto, A. Itoh, A. Kirilyuk, and T. Rasing, and A. V. Kimel, Nature (London) 472, 205 (2011).

${ }^{8}$ T. A. Ostler, J. Barker, R. F. L. Evans, R. W. Chantrell, U. Atxitia, O. Chubykalo-Fesenko, S. El Moussaoui, L. Le Guyader, E. Mengotti, L. J. Heyderman, F. Nolting, A. Tsukamoto, A. Itoh, D. Afanasiev, B. A. Ivanov, A. M. Kalashnikova, K. Vahaplar,
J. Mentink, A. Kirilyuk, T. Rasing, and A. V. Kimel, Nature Commun. 3, 666 (2012).

${ }^{9}$ J. H. Mentink, J. Hellsvik, D. V. Afanasiev, B. A. Ivanov, A. Kirilyuk, A. V. Kimel, O. Eriksson, M. I. Katsnelson, and T. Rasing, Phys. Rev. Lett. 108, 057202 (2012).

${ }^{10}$ U. Atxitia, P. Nieves, and O. Chubykalo-Fesenko, Phys. Rev. B 86, 104414 (2012).

${ }^{11}$ T. A. Ostler, R. F. L. Evans, R. W. Chantrell, U. Atxitia, O. Chubykalo-Fesenko, I. Radu, R. Abrudan, F. Radu, A. Tsukamoto, A. Itoh, A. Kirilyuk, T. Rasing, and A. Kimel, Phys. Rev. B. 84, 024407 (2011).

${ }^{12}$ U. Atxitia, O. Chubykalo-Fesenko, J. Walowski, A. Mann, and M. Münzenberg, Phys. Rev. B 81, 174401 (2010).

${ }^{13}$ M. Sultan, U. Atxitia, A. Melnikov, O. Chubykalo-Fesenko, and U. Bovensiepen, Phys. Rev. B 85, 184407 (2012).

${ }^{14}$ D. A. Garanin, Phys. Rev. B 55, 3050 (1997).

${ }^{15}$ O. Chubykalo-Fesenko, U. Nowak, R. W. Chantrell, and D. Garanin, Phys. Rev. B 74, 094436 (2006).

${ }^{16}$ U. Atxitia and O. Chubykalo-Fesenko, Phys. Rev. B 84, 144414 (2011).

${ }^{17}$ R. F. L. Evans, D. Hinzke, U. Atxitia, U. Nowak, R. W. Chantrell, and O. Chubykalo-Fesenko, Phys. Rev. B 85, 014433 (2012). 